\title{
PARÁSITOS PRESENTES EN Periplaneta americana LINNAEUS "CUCARACHA DOMÉSTICA" DE LA CIUDAD DE ICA
}

\author{
PARASITES OF Periplaneta americana Linnaeus "DOMESTIC COCKROACH" \\ FROM ICA \\ Mary Fernández B.', Diana M. Martínez M.', Manuel Tantaleán V. 'y \\ Rosa Martínez R.3
}

\section{RESUMEN}

Se estudiaron 244 especimenes de Periplaneta americana Linnaeus procedentes del alcantarillado de 13 zonas de la ciudad de lca. Se identificaron 2 protozoarios y 2 nemátodes propios de la fauna parasitaria de l: "cucaracha doméstica": Lophomonas blattarum, Leptomonas sp., Leidynema appendiculatum y Hammerschmidtiella diesingi y protozoarios que parasitan al hombre, de los cuales 3 especies son patógenas: Giardia lamblia, Blastocystis hominis y Cryptosporidium $\mathrm{sp}$.

Palabras clave: Parásitos, Periplaneta americana, Perú.

\section{SUMMARY}

244 specimens of Periplaneta americana Linnaeus from 13 localities of I ca were studied. Nematodes and protozoa were identified. They are Lophomonas blattarum, Leptomonas sp., Leidynema appendiculatum and Hammerschmidtiella diesingi. Also, parasites of man were found, three of which are pathogenic: Giardia lamblia, Blastocystis hominis and Cryptosporidium sp.

Keywords: Parasites, Periplaneta americana, Peru.

\section{INTRODUCCIÓN}

Periplanela americana Linnaeus, "cucaracha doméstica", es un insecto ortóptero que tiene su propia fauna parasitaria y al mismo tiempo es huésped intermediario de algunos helmintos de vertebrados (Ramírez, 1989) pero que, al vivir en contacto con material orgánico en descomposición proveniente del sistema de alcantarillado de las viviendas y de las industrias, está en intima relación con otros organismos patógenos como quistes de protozoarios y huevos de helmintos que permanecen viables tanto en el tegumento como

\footnotetext{
1 Departamento de Ciencias Biologicas, Facultad de Ciencias, Universidad Nacional San Luis Gonzaga de Ica. Ica, Perú

2 Departamento de Microbiologia, Facultad de Ciencias, Universidad Peruana Cayetano Heredia.

3 Departamento de Microbiologia y Parasitología, Facultad de Ciencias Biológicas. UNMSM. e-mail: d190033@unmsm.edu.pe
}

en el intestino. Por esta razón, es un importante vector mecánico y transmisor de microorganismos que afectan la salud del hombre y de los animales domésticos porque contamina los alimentos con sus patas o excrementos o con sus vómitos. La alternancia de hábitat durante el día y la noche los convierte en insectos contaminadores peligrosos ai desplazarse en las cocinas y comedores de domicilios, almacenes y aun de hospitales.

En el Perú, son escasos los trabajos sobre la fauna parasitaria propia de las "cucarachas" (De la Cruz y Tantaleán, 1980; Iannacone et al., 1999) y aquellos que tratan de los parásitos de importancia médica humana que son vectorizados por estos insectos. Es nula la bibliografía para la ciudad de Ica a pesar de la relación de este insecto con algunos parásitos patógenos como Cryplosporidium (Zerpa y Huicho, 1994).

El presente trabajo tiene por finalidad iden tificar los parásitos propios de Periplaneta 
Tabla 1.Prevalencia de infección por especie de protozoos en Periplaneta americana de la localidad de Ica.

\begin{tabular}{rccc}
\hline LOCALIDAD & N. ${ }^{\circ}$ DE & \multicolumn{2}{c}{ CUCARACHAS PARASTADAS } \\
(ZONAS) & CUCARACHAS & N. $^{\circ}$ & $\%$ \\
\hline San Isidro & 20 & 15 & 75,00 \\
Divino Maestro & 24 & 21 & 87,50 \\
Sta. Rosa del Palmar & 4 & 4 & 100,00 \\
Sto. Domingo & 8 & 7 & 87,50 \\
Moderna & 8 & 3 & 37,50 \\
San Miguel & 24 & 4 & 16,67 \\
Pedreros & 24 & 17 & 70,83 \\
La Palma & 28 & 19 & 67,86 \\
Cercado & 48 & 33 & 68,75 \\
Sol de Ica & 4 & 11 & 25,00 \\
San José & 16 & 1 & 68,75 \\
Cementerio & 20 & 16 & 80,00 \\
V. Maurtua & 16 & 13 & 81,25 \\
TOTAL & 244 & 164 & 67,21 \\
\hline
\end{tabular}

americana de la ciudad de Ica, determinar aquellos que podrian afectar la salud del hombre y establecer su prevalencia.

\section{MATERIAL Y MÉTODOS}

Durante los meses de febrero y agosto de 1997 se capturaron 244 especímenes de Periplaneta americana de los cuales, en razón de la metodología empleada, sólo a 180 se les investigó protozoarios propios de su fau- na y elementos parasitarios que infectan al hombre. Los insectos se capturaron de las alcantarillas de los domicilios en las siguientes zonas del cercado de la ciudad de Ica: San Isidro, Divino Maestro, Santa Rosa del Palmar, Santo Jomingo, La Moderna, San Miguel, Pedreros, La Palma, Cercado, Sol de Ica, San José, Cementerio y V. Maurtua. Todos los datos obtenidos se anotaron en una ficha previamente preparada. Los insectos se trasladaron al laboratorio para procesarlos. Los pará-

Tabla 2.Prevalencia de infección por especie de protozoos en Periplaneta americana de la localidad de Ica.

\begin{tabular}{cccc}
\hline N. ${ }^{\circ}$ & \multicolumn{2}{c}{ CUCARACHAS } \\
CUCARACHAS & PROTOZOOS & \multicolumn{2}{c}{ PARASITADAS } \\
& & N. ${ }^{\circ}$ & $\%$ \\
\hline \multirow{2}{*}{180} & Lophomonas blattarum & 5 & $2, x$ \\
& Leptomonas sp. & 2 & 1,1 \\
TOTAL & & 7 & 4,0 \\
\hline
\end{tabular}


Tabla 3.Prevalencia de infección por especie de nematodes en Periplaneta americana de la ciudad de lca.

\begin{tabular}{cccc}
\hline \multirow{2}{*}{ N.DDE } & HELMINTOS & \multicolumn{2}{c}{ CUCARACHAS } \\
CUCARACHAS & PARASTTADAS \\
& & N. ${ }^{\circ}$ & $\%$ \\
\hline \multirow{2}{*}{244} & Hammerschmidtiella diesingi & 30 & 12,3 \\
& Leidynema appendiculatum & 52 & 21,3 \\
\hline
\end{tabular}

sitos se obtuvieron provio sacrificio de los insectos con éter.

Los quistes y huevos adheridos al tegumento su colectaron lavando los insectos con suero fisiológico estéril y centrifugado a $2000 \mathrm{rpm}$ durante 3 minutos. Una porción del sedimento se coloreó con lugol y se observó directamente en el microscopio y la otra se fijó con alcohol polivinílico sobre una lámina portaobjetos que luego se coloreó con la téc- nica iricrómica de Gomori modificada por Cimyoun para investigar la presencia de ooquistes de Cryptosporidium

Los parásitos, quistes y huevos del intestino se colectaron previa disección y exposición del órgano; en primer lugar se recogieron los nemátodes, que se lavaron y fijaron en alcohol etílico de $70 \%$ caliente; luego con el contenido intestinal se procedió como en el caso de la colección de los parásitos del tegumento.

Tabla 4.Prevalencia de infección por nematodes de Periplaneta americana en la ciudad de Ica.

\begin{tabular}{rccc}
\hline LOCALIDAD & \multirow{2}{*}{${ }^{\circ}$ DE } & & \multicolumn{2}{c}{ CUCARACHAS PARASITADAS } \\
(ZONAS) & CUCARACHAS & N. ${ }^{\circ}$ & $\%$ \\
\hline San Isidro & 20 & 15 & 75,00 \\
Divino Maestro & 24 & 21 & 87,50 \\
Sta. Rosa del Palmar & 4 & 4 & 100,00 \\
Sto. Domingo & 8 & 7 & 87,50 \\
Moderna & 8 & 3 & 37,50 \\
San Miguel & 24 & 2 & 8,33 \\
Pedreros & 24 & - & $\cdots$ \\
La Palma & 28 & - & $\cdots$ \\
Cercado & 48 & 9 & 18,75 \\
Sol de Ica & 4 & 1 & 25,00 \\
San Jose & 16 & 4 & 25,00 \\
Cementerio & 20 & 7 & 35,00 \\
V. Maurtua & 16 & 4 & 25,00 \\
TOTAL & 244 & 77 & 31,55 \\
\hline
\end{tabular}


La identificación de los nemátodes se hizo previa clarificación en una mezcla de alcohol-fenol.

\section{RESULTADOS}

Al estudiar 244 especímenes de Periplaneta americana se encontraron los siguientes parásitos: 2 especies de protozoarios y 2 helmintos que son parte de la fauna natural y 7 especies de protozoarios que el insecto ha adquirido en su hábitat y que correspondan a parásitos del hombre; de este modo la "cucaracha" se comporta como un vector mecánico. Dentro de este grupo de parásitos, algunas especies son patógenas y por lo tanto de importancia en salud humana.

La siguiente lista de parásitos o elementos parasitarios encontrados en Periplaneta americana resume los hallazgos en la ciudad de Ica:

\section{A) Parásitos de la fauna natural}

Protozoarios

- Lophomonas blattarum (Mastigophora, Lophomonadidae)

-Lepromonas sp. (Mastigophora, Trypanosomatidae)

\section{Helmintos}

- Hammerschdmidtiella diesigni (Nematoda, Thelastomatidae)

- Leidynema appendiculatum (Nematoda, Thelastomatidae)

\section{B) Parásitos del hombre}

Protozoarios

- Giardia lamblia (Mastigophora, Hexamitidae)
- Endolimax nana (Sarcodina,

Entamoebidae)

- Eniamoeba coli (Sarcodina,

Entamoebidae)

- Chilomastix mesnili (Mastigophora, Retortamonadidae)

- Iodamoeba bütschlii (Sarcodina, Entamoebidae)

- Blastocystis hominis (Sarcodina, Blastocystida)

-Cryptosporidium sp. (Coccidia, Cryptosporidiidae)

A pesar de haberlos buscado en la cavidad corporal del artrópodo, no se encontraron cistacantos de Moliniformis moliniformis, un acantocéfalo que parasita a las ratas y al hombre (Moore et al., 1994).

De la lista anterior, los helmintos, aun cuando se han encontrado en algunas localidades del Perú, no eran conocidos en Ica.

A continuación se describen brevemente Lophomonas blattarum y Leptomonas sp. porque son los únicos protozoarios que son reportados por primera vez en el Perú.

\section{Lophomonas blattarun Stein, 1859}

Flagelado Lophomonadidae de cuerpo ovalado o piriforme, de 25 a 30 micras de longitud; con numerosos flagelos a manera de manojo situados en el extremo anterior, siendo de mayor tamaño aquellos que se encuentran distantes de la cisura apical. El protoplasto lleva numerosas vacuolas alimenticias, un núcleo que se localiza anteriormente y el axostilo que se proyecta fuera del cuerpo.

\section{Leptomonas sp.}

Flagelado Trypanosomatidae de cuerpo alargado con extremo anterior afilado. Con flagelo que nace del extremo anterior de un 
Tabla 5. Prevalencia de infección de Periplaneta americana como vector mecánico de protozoarios parásitos del hombre.

\begin{tabular}{|c|c|c|c|}
\hline \multirow{2}{*}{$\begin{array}{c}\text { NODE } \\
\text { CUCARACHAS }\end{array}$} & \multirow{2}{*}{ PROTOZOARIOS } & \multicolumn{2}{|c|}{$\begin{array}{l}\text { CUCARACHAS } \\
\text { PARASITADAS }\end{array}$} \\
\hline & & N.o & $\%$ \\
\hline \multirow{7}{*}{180} & Giardia lomblia & 4 & 2,2 \\
\hline & Endolimux narta & 24 & 13,3 \\
\hline & Entamoeba coli & 3 & 2 \\
\hline & Chilomastix mesnili & 6 & 3,3 \\
\hline & lodamoeba bütschilii & 1 & 0,5 \\
\hline & Blastocystis hominis & 88 & 49 \\
\hline & TOTAL & 126 & 70 \\
\hline
\end{tabular}

blefaroplasto y cuya longitud es aproximadamente 3 veces el largo del cuerpo. Miden en total de 31,5 a 50,4 micras de longitud, pero la longitud promedio del cuerpo es de 10,29 y la del flagelo 29,26 micras. El núcleo se sitúa ligeramente excéntrico.

La Tabla 1. nos muestra la prevalencia de infección por protozoarios y/o helmintos en 244 "cucarachas" de 13 zonas de la ciudad de Ica, donde el $67,21 \%$ estuvieron parasitadas.

La Tabla 2 referente a protozoos propios de la "cucaracha" nos permite apreciar que sólo el 4\% de 180 "cucarachas" presentaron infección por Lophomonas blattarun o Leptomonas sp., siendo la prevalencia de la primera de $2,8 \%$ y de la segunda $1,1 \%$.

La tabla 3 nos muestra el parasitismo por nemátodes, en la cual se ve que es mayor la prevalencia por Leidynema appendiculatum $(21,31 \%)$. Este helminto es cosmopolita y puede parasitar a otros miembros de la familia Blattidae.
La tabla 4 nos permite conocer que el $31,55 \%$ de 244 "cucarachas" presentaron infección por nemátodes, así como las localidades más afectadas: Sta. Rosa del Palmar (100\%), Sto. Domingo $(87,5 \%)$, Divino Maestro $(87,5 \%)$ y San Isidro $(75 \%)$.

Los quistes y trofozoítos de parásitos que infectan al hombre se encontraron en el $70 \%$ de 180 "cucarachas", como se muestra en la tabla 5 , donde la mayor prevalencia corresponde a Blastocystis hominis (49\%).

Las tablas 6, 7 y 8 nos muestran respectivamente la infección por Cryptosporidium sp., la distribución de los parásitos encontrados ya sea en el tegumento o el intestino y la prevalencia e intensidad de infección de las especies de nemátodes. Esta última tabla nos muestra que el máximo número de $L$. appendiculatum en un artrópodo fue de 17, algo excepcional, pues normalmente oscila entre 1 y 6.

Tabla 6. Infección de Periplaneta americana por Cryptosporidium sp. en la ciuviad de Ica.

\begin{tabular}{ccc}
\hline N.oDE & PARÁsITOS & $\%$ \\
CUCARACHAS & & \\
\hline 20 & 4 & 20,00 \\
\hline
\end{tabular}




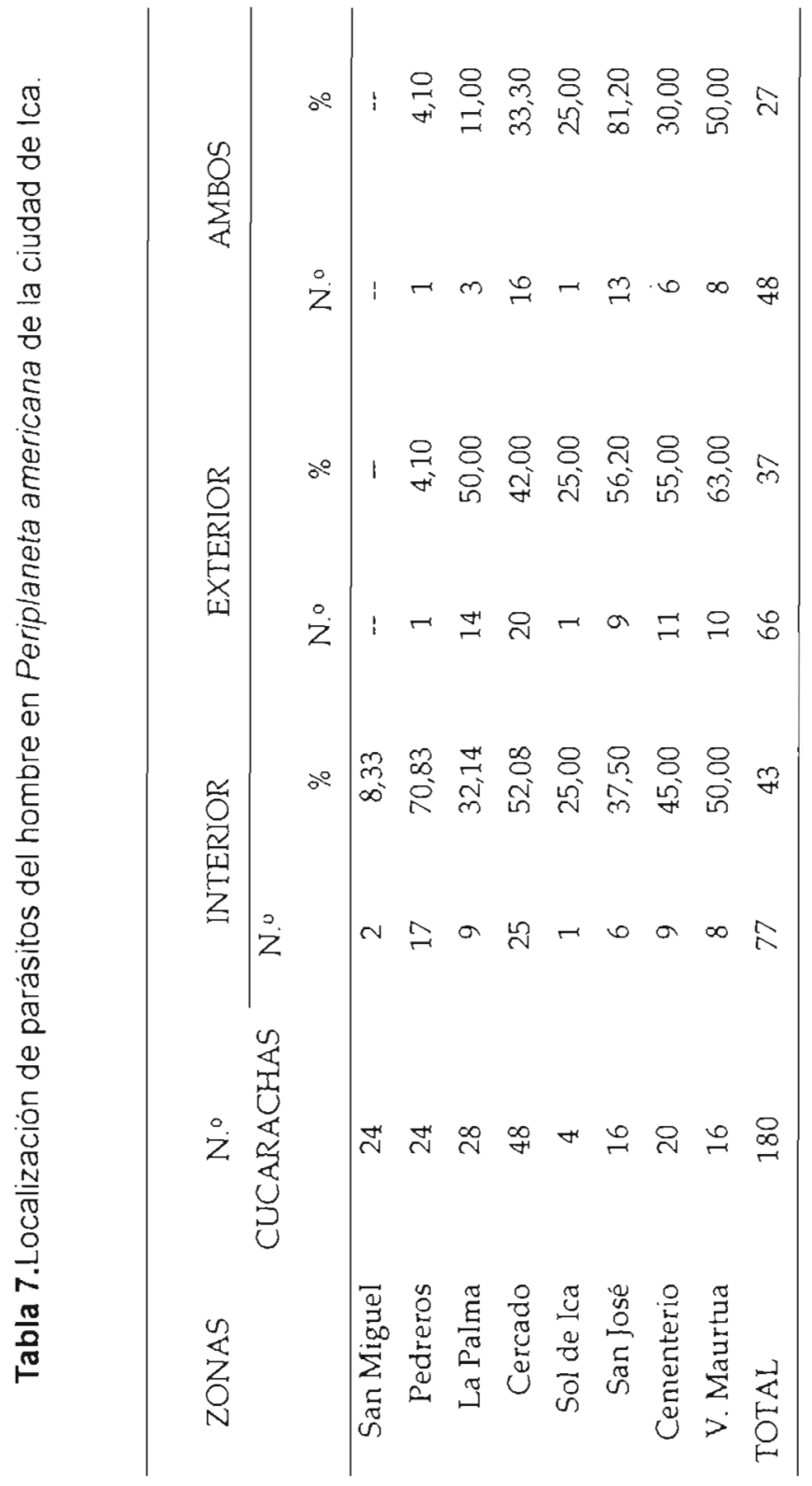




\section{DISCUSIÓN}

En el Perú son muy pocos los trabajos relacionados con los parásitos que infectan a Periplaneta americana a pesar de la importancia de estos insectos. La mayoría de los artículos se relacionan con los parásitos del hombre donde la "cucaracha" juega un rol importante como vector mecánico.

El presente trabajo ha investigado a 244 especímenes de $P$. americana de la ciudad de lca no sólo en su condición de transportadores de elementos parasitarios que infectan al hombre sino también por sus parásitos que constituyen lá fauna natural, dentro de los cuales los protozoarios eran desconocidos.

Dos protozoarios flagelados son los primeros hallazgos en el Perú: Lophomonas blattaurm y Leptomonas sp. La primera es una especie cosmopolita que puede parasitar a otros miembros de la familia Blattidae; sus características permiten reconocerla con facilidad; no es patógena y su prevalencia en Ica alcanza el 2,8\%.

La Lepiomonas sp. que encontramos presenta caracteristicas que no coinciden con las descripciones de las especies reportadas en otros artrópodos por lo que es el primer reporte hecho para Periplanela. Nuestros especimenes tienen flagelo muy largo, cuya longitud alcanza casi 3 veces la longitud del cuerpo; este detalle los diferencia de todas las especies conocidas; por esta razón no le hemos asignado un nombre especifico; esperamos obtener nuevo material que nos permita un estudio más detallado y poder realizar la identificación específica. Sólo el 1, 1\% de las "cucarachas" estudiadas lo presentaron.

Los nemátodes que encontramos, Leydinema appendiculatun y Hammerschmidtiella diesingi ya eran conocidos en el Perú (De la Cruz, 1976; De la Cruz y Tantaleán, 1980; Iannacone et al., 1999). Estas especies son cosmopolitas y viven en el intestino posterior del huésped sin causar daño alguno (Kloss. 1966). La primera especie se encontró en todas las "cucarachas" de la localidad de Sta. Rosa del Palmar, con un promedio de 3 gusanos por insecto. De acuerdo a McCallister (1988), cuando existe infección por 2 especies en el mismo individuo huésped y una de las cuales es $H$. diesingi, el número de individuos de esta especie en el intestino tiende a ser menor.

Ambas especies no afectan al hombre, pero como los huevos son similares a los de Enterobius vermiculuris (un gusano común en los niños del Perú), podrían confundirse si no se toman en cuenta los detalles morfométricos cuando se encuentrin huevos de oxyuroideos en ambientes y objetos durante el desarrollo de estudios epidemiológicos sobre enterobiosis, como lo anotan Du la Cruz y Tantaleán (1980).

$P$. americana es un importante vector mecánico de numerosos microorganismos que infectan al hombre, como lo señalan Cochran (1982) y Ramírez (1989), y junto con las moscas contaminan los alimentos (Miranda y Martinez, 1997: Zárate y Mejía, 1995) debido a sus hábitos de posarse sobre la materia orgánica en descomposición como las letrinas, desagües y basurales.

Villanueva el al. (1988) y Villanueva et a' (1995) han demostrado que, después de 20 años, el $80 \%$ de niños entre l y 15 años de la población rural y urbana de la ciudad de lca estaban altamente parasitados y sin ningún cambio en la prevalencia parasitaria. En parte, esto se explica por la presencia de los vectores mecánicos como las "cucarachas".

De las 7 especies de protozoarios que infectan al hombre y que encontramos en $P$. americana, 3 son patógenas: Giardia lamblia, Blastocystis hominis y Cryplosporidium sp. $B$. hominis no había sido reportada en ningún trabajo previo en $P$. americana por lo que constituye el primer hallazgo; se encuentra en el intestino del $49 \%$ de los insectos investigados. Esta ameba puede producir diarrea dependiendo del número de parásitos presentes 
Tabla 8. Prevalencia intensidad de infección por 2 especies de nemàtodes en la ciudad de lca H. d: Hammerschamidtiella diesigni y L. a: Leidynema appendiculatum

\begin{tabular}{|c|c|c|c|c|c|c|}
\hline \multirow[t]{2}{*}{ ZONAS } & \multicolumn{2}{|c|}{$\begin{array}{l}\text { NEMATODES N/N } \\
\left({ }^{*}\right)\end{array}$} & \multicolumn{2}{|c|}{$\begin{array}{l}\text { PREVALENCIA } \\
\%\end{array}$} & \multicolumn{2}{|c|}{$\begin{array}{l}\text { INTENSIDAD } \\
\text { (RANGO) }\end{array}$} \\
\hline & L. a & H.d. & L. $a$ & H.d & L. $\bar{a}$ & H.d \\
\hline San Isidro & $20 / 10$ & $20 / 9$ & 50.0 & 45.0 & $2.9(1-6)$ & $2.6(1-7)$ \\
\hline Divino Maestro & $24 / 14$ & $24 / 8$ & 58.3 & 33.3 & $4.2(1-17)$ & $1.0(1-6)$ \\
\hline Sta. Rosa del Palmar & $4 / 4$ & $4 / 0$ & 100.0 & 0 & $3.0(1-4)$ & 0 \\
\hline Sto. Domingo & $8 / 5$ & $8 / 2$ & 7.3 & 25.0 & $1.8(1-4)$ & $1.5(1-2)$ \\
\hline Moderna & $8 / 3$ & $8 / 0$ & 38.0 & 0 & $2.6(1-5)$ & 0 \\
\hline San Miguel & $24 / 1$ & $24 / 1$ & 4.2 & 4.2 & $4.0 \quad(4)$ & $1.0(1)$ \\
\hline Pedreros & $24 / 0$ & $24 / 0$ & 0 & 0 & 0 & 0 \\
\hline La Palma & $28 / 0$ & $28 / 0$ & 0 & 0 & 0 & 0 \\
\hline Cercado & $48 / 7$ & $48 / 2$ & 15.0 & 4.2 & $2.3(1-4)$ & $4.0(1-5)$ \\
\hline Sol de Ica & $4 / 0$ & $4 / 1$ & 0 & 25.0 & 0 & $2.0 \quad(2)$ \\
\hline San José & $16 / 2$ & $16 / 2$ & 13.0 & 13.0 & $2.0(1-3)$ & $2.0(1-3)$ \\
\hline Cementerio & $20 / 4$ & $20 / 3$ & 20,0 & 15.0 & $0.5(1-2)$ & $3.3(1-4)$ \\
\hline V. Maurtua & $16 / 2$ & $16 / 2$ & 13.0 & 13.0 & $3.0(1-3)$ & $1.5(1-2)$ \\
\hline
\end{tabular}

* N/N: Numero de cucarachas estudiadas sobre número de cucarachas infectadas según especie.

y del estado del paciente (Stenzel y Boreham, 1996; Zierdt, 1991).

G. lamblia es un protozoario que también produce diarrea en el 23,76\% de los niños de la ciudad de Ica (Villanueva et al,, 1988).

Cryptosporidium sp. es un parásito de ciclo de vida directo, cuya forma infectante es el ooquiste maduro; es un patógeno imporlamle cue produce diarrea principalmente en "niños y en personas inmunodeprimidas a los que puede llevar a la muerte (Fayer y Ungar, 1986). Fn el Peru, Zerpa y' Huicho( 1994) han reportado el hallazco de dos "cucarachas" infectadas con este coccidio. Nuestro hallazgo en Ica reviste importancia porque el insecto vector abandona su refugio durante la noche y se posa subre los alimentos humanos a los que contamina con sus patas, heces y vómito. Lamentablemente, no se han realizado estudios para conocer la prevalencia de
Cryplosporidizm sp. en las "cucarachas" no sólo de la ciudad de lca sino de otras localidades del Perú.

\section{LITERATURA CITADA}

Cochranı, D. G. 1982. Cockroaches. Biology and control. Documento de la Organización Mundial de la Salud. W/lO/VBC/82.856 pp. Ginebra.

De l.al Cru\%. M. 13. 1976. Acmátodos parásitos Je aleunos Blattidae (Orthoptera) en el Perú. Tesis Titulo Profesional de Biólogo. Universidad Ricardo Palina. I.ima.

De La CruL. B. M. \M. Tantaleán. 1980. Estudio k algunos nemálodos de "cucarachas" del P’eru Bol. Periano Parasit. 2: $51-56$.

l'ilyer, R. and B.i.p. Ungar. 1986. (ryplosporidium sp. and cryplosporidiosis. Micrubiolonical Review, 50: 458-48.3

Jannacone J.; K. Velásquez y A. Arrascus. 1999. Fauna parasitaria de Periplaneta amerticana Linnaeus in un distrito de Lima. Rev. Per. Biol. 6: 54-60. 
Kloss, G.r. 1966. Revisão dos nematoides de Blattaria do Brasil. Papêis Avulsos Dep. Zool Sào Paulo, 18: 147-188

Mccallister, G. L. 1988. The effect of Thelastoma bulhoesi and Hammerschmidtiella diesingi (Nematoda: Oxyurata) on host size and physiology in Periplanela americana (Arthropoda: Blattidae). Proc. Helminthol. Soc. Wash., 55: 12-14.

Miranda, E. I. y R. R. Martínez. 1997. Estudio preliminar sobre la importancia de las moscas como transmisores mecánicos de ooquisles de Cryplosporidium sp. Lib. Res. VI Reunión Cientifica det ICBAR. UNMSM. Lima. Res. 84

Moore, J., Freehling, M. and J, Gotelli. 1994. Altered behavior in two species of blattid cockroaches infected with Moniliformis moniliformis (Acanthocephala). J. Parasitol., 80: 220-223.

Ramírez, P. J. 1989. La cucaracha como vector de agentes patógenos. Bol. Oficina San. Panam., 107: $41-53$.

Stenzel, D. J. and P. F. L. Boreham. 1996. Blastocystis hominis revised. Clin. Microbiol. Review, 9: $563-584$
Villanueva, R. C.; M. E. Córdova; B. A. Maldonado y B. O. Mendoza. 1988. Evaluación después de 20 años de la enteroparasitosis en la población infantil ( 1 - 15 años) de los pueblos jóvenes de la ciudad de Ica. Lib. Res. Simposium Parasitismo Intestinal en el Hombre. Lima. Res. 25.

Villanueva. R. C.; P. M. Hidalgo y T. N. Lovera. 1995. Prevalencia de parasitismo intestinal en la provincia de [ca hasta el año 1988. Lib. Res. II Cong. Peruano Parasil. 'Trujillo. Res. 16

Zárale, A. M. y D. E. Mejía. 1995. Frecuencia de enteroparásitos del hombre portados por Musca domestica y Periplanela americana en Trujillo. I,ib. Res. II Cong. Peruano Parasit., Trujillo. Res. 12

Zerpa, R. and L. Huicho. 1994. Chilhood cryptosporidial diarrhea associated with identification of Cryplosporidium in the cockroach Periplaneta americana. Ped. Inf. Dis. J., 13: 546-548.

Zierdt, Ch. H. 1991. Blaslocystis hominis, past and fulure. Clin. Microbiol. Review. 4: 61-79. 